\title{
Empresarios, ambiente, territorio y redes. Las nociones culturales y su construcción social $^{1}$
}

\author{
Juan Carlos Ruíz Urquijo
}

Recibido: 18 de julio de 2013

Aprobado: 30 de agosto de 2013

\section{Ruíz, J. C. (2013). Empresarios, ambiente, territorio y redes. Las nociones culturales y su construcción social. Activos 21, 43-61}

Clasificación JEL: Q 00, Q 20

\section{Resumen}

El documento construye una reflexión sobre la relación entre cultura organizacional, problemas ambientales y construcción de redes, a partir de un marco teórico celaborado con teorías organizacionales, geográficas y sociológicas, en el que se busca develar la relacion entre la creación de redes y las nociones culturales de los empresarios, enfatizándo en un fenómeno crítico de la sociedad actual: el problema ambiental, estructurado desde aspectos de responsabilidad social empresarial.

El trabajo se compone de tres apartados, la contextualización del empresario en la estructura moderna, los fenómenos culturales de los

1 El documento es producto del proyecto de Investigación denominado Percepciones de RSE de empresarios MiPyme Bogotanos, del Programa de Contaduría Pública de la Fundación Universitaria Agraria de Colombia, iniciado en el segundo semestre de 2013.

2 Contador Público Universidad Nacional de Colombia, (c) Mg en Desarrollo Sustentable y Gestión Ambiental Universidad Distrital Francisco José de Caldas, Especialista en gerencia de Recursos Naturales Universidad Distrital Francisco José de Caldas. Docente Fundación Universitaria Agraria de Colombia, Sede Bogotá. 
empresarios y la noción de red y territorio recreadas a la luz de los dos anteriores, lo que permitirá expresar desde las redes, su construcción como estructuras socio-técnicas, con base en la generación de alianzas entre los empresarios y sus partes interesadas.

\title{
Palabras clave
}

Responsabilidad social empresarial, redes, territorio.

Ruíz, J. C. (2013). Businesmen, environment, land and networks. Cultural notions and your social construction. Activos 21, 43-61

\begin{abstract}
This paper makes a reflection on the relationship between organizational culture, environmental and building networks, since a theoretical framework from organizational, geographical and sociological theories, which seeks to uncover the relating between networking and cultural notions of entrepreneurs, emphasizing a critical issue in today's society: the environmental problem from structured aspects from corporate social responsibility (CSR).

This document consists in three sections ranging from the contextualization of the entrepreneur in the modern structure, the cultural phenomena of entrepreneurs and the notion of network and recreated in the light of the two previous territory, allowing express from building networks the same as socio-technical structure from the creation of partnerships between entrepreneurs and their stakeholders.
\end{abstract}

\section{Keywords}

Corporate Social Responsibility, Networks, Territory. 
Ruíz, J. C. (2013). Entrepreneurs, environnement, territoire et réseaux. Les notions culturelles et leur construction sociale. Activos 21, 43-61

\section{Résumé}

Le document construit une réflexion sur la relation entre culture organisationnelle, problèmes environnementaux et construction de réseaux, à partir d'un cadre théorique établi à partie de théories organisationnelles, géographiques et sociologiques, dans le but de dévoiler la relation entre la création de réseaux et les notions culturelles des entrepreneurs, en mettant l'accent sur un phénomène critique de la société actuelle : la problématique environnementale, structurée sous les aspects de responsabilité sociale d'entreprise.

Le travail est composé de trois paragraphes, la contextualisation de l'entrepreneur dans la structure moderne, les phénomènes culturels des entrepreneurs et la notion de réseau et territoire, reconstitués au regard des deux antérieures, ce qui permettra d'exprimer depuis des réseaux, sa construction en tant que structures sociotechniques, sur la base de la création d’alliances entre les entrepreneurs et leurs parties intégrales.

\section{Mots clés}

Responsabilité sociale d’entreprise, réseaux, territoire. 


\section{Introduction}

Los factores ambientales son un paradigma dentro de las organizaciones empresariales y motivan la creación de estrategias, para su manejo, que develan una pregunta ulterior: ¿qué estructuras discursivas poseen los empresarios sobre los aspectos ambientales que tocan sus organizaciones?

A partir de este cuestionamiento y en el marco de la investigación sobre responsabilidad social en Mipymes se genera este texto. Reflexionando sobre ¿cuáles son los principios culturales que recrean los comportamientos empresariales? Se observa la estructura cultural de las organizaciones para vincularla con los conceptos redes y territorio, mirada que desde la noción geográfica determinará estructuras de localización para establecer estructuras de innovación ante el paradigma ambiental. Así, la relación expuesta será el propósito de desarrollo del documento, el cual se dividirá en tres partes: la contextualización del empresario en la estructura moderna, los fenómenos culturales de empresarios y las nociones de red y territorio recreadas a la luz de las dos anteriores.

\section{La empresa dentro de la modernidad}

En la sociedad actual la empresa tiene un papel dominante que conlleva a factores de desarrollo social, político y económico permitiendo un arraigo cultural en el medio que interactúa y diferenciándose por su formación, características económicas, leyes que lo rigen, afectación hacía su entorno, lugar donde se encuentra, etc. Estas son características propias de cada empresa que evolucionan a través de la historia, pero ¿cuál es el origen de las empresas en la historia y cuáles son sus principales particularidades de desempeño dentro de la sociedad? Para responder esta pregunta se analizará la tabla 1 elaborada con datos construidos desde Prieto (2008), quien se enfoca en cinco épocas del tiempo: 
Tabla 1. Principales características de la empresa a lo largo de la historia

\begin{tabular}{|c|c|c|}
\hline Época & Características principales & Aporte a la sociedad \\
\hline \multirow{4}{*}{ Primitiva } & $\begin{array}{l}\text {-Actividades de pesca, caza, y } \\
\text { recolección }\end{array}$ & $\begin{array}{l}\text {-Jefe de familia como } \\
\text { autoridad }\end{array}$ \\
\hline & -No lucrativas & -Trabajo en grupo \\
\hline & -Comercio doméstico & \\
\hline & -Pago con comida y vivienda & \\
\hline \multirow{3}{*}{ Esclavista } & -Patrón, esclavos & -Individuo como \\
\hline & -Trabajo doméstico y en la tierra & mercancía de intercambio \\
\hline & -Lucro & -Castigo hacia el esclavo \\
\hline \multirow{6}{*}{ Feudalista } & -Servidumbre & -Desplazamiento del \\
\hline & -Control de la producción & individuo \\
\hline & -Pago con porciones de tierra & -Trabajo en familia \\
\hline & -Aumento de capital del feuda & -Descentralización del \\
\hline & -Empresa artesanall & \\
\hline & -Intercambio comercial & $\begin{array}{l}\text {-Organización de la } \\
\text { propiedad }\end{array}$ \\
\hline \multirow{7}{*}{ Industrialización } & -Tecnificación & -Desarrollo urbano \\
\hline & -Privatización de la empresa & -Centralización de la \\
\hline & -Crecimiento industrial & familias en las grandes \\
\hline & -Incremento de la producción & t \\
\hline & -Lucro & $\begin{array}{l}\text {-Aumento de la mano de } \\
\text { obra mal pagada }\end{array}$ \\
\hline & $\begin{array}{l}\text {-Aumento de los beneficios } \\
\text { económicos para los } \\
\text { empresarios }\end{array}$ & $\begin{array}{l}\text {-Diversificación de la } \\
\text { cultura }\end{array}$ \\
\hline & & $\begin{array}{l}\text {-Empresa como eje central } \\
\text { de la sociedad }\end{array}$ \\
\hline \multirow{5}{*}{ Capitalista } & -Lucro & -Individualismo \\
\hline & -Utilización desmesurada del & -Crecimiento demográfico \\
\hline & medio ambiente & -Creación de necesidades \\
\hline & $\begin{array}{l}\text {-Dominio del empresario, el } \\
\text { productor y los financistas }\end{array}$ & $\begin{array}{l}\text {-Tránsito de trabajadores } \\
\text { artesanales a }\end{array}$ \\
\hline & -Calidad en la producción & consumidores impulsivos \\
\hline
\end{tabular}

Fuente: elaboración propia con datos de Prieto (2008) y Dupuis (2010). 
Como se puede observar en la tabla 1, la época primitiva se caracterizó por un comercio doméstico o, según Solomon (1995), oikonomikos, en dónde el manejo empresarial era de dominio estrictamente familiar. Sin darse cuenta estos primitivos dieron origen al concepto de empresa, enmarcada por la supervivencia y no como un fin lucrativo. Luego vino la época esclavista y feudal que se diferenciaba por el comercio de esclavos, en esta se inició el crecimiento industrial y el desplazamiento del campesino a las grandes ciudades; el lucro se fue convirtiendo en el principal generador de empresa y en la principal causa del desplazamiento del campesino (Prieto, 2008).

En la época de la industrialización se logra aseverar que

esta transformación puede explicarse, en parte, en términos de desarrollo urbano, de sociedades mayores y más centralizadas, de la privatización de los grupos familiares como consumidores, del rápido progreso tecnológico y del crecimiento de la industria y el desarrollo asociado de las estructuras necesidades y deseos sociales (Solomón 1995, p. 485).

En este sentido se convierte en chrematisike o comercio para el lucro (Solomón, 1995) y la empresa pasa a ser el eje central de la sociedad. Dada la revolución industrial en el siglo XIX y gracias a los grandes descubrimientos que permitieron transformaciones para las empresas, estas pasan de lo artesanal o lo industrial, de lo manual a la maquinaria. Dichos procesos implican cambios en el concepto de empresa y de la influencia que ejerce en la sociedad, convirtiéndola en una comunidad capitalista donde lo más importante para el empresario es el lucro (Dupuis, 2010), la maximización de sus riquezas (paradigma que aún influye en nuestro empresario y en nuestra sociedad).

Este crecimiento desmedido de la empresa con fines de lucro y las necesidades creadas en los últimos tiempos, hacen que se utilicen de forma indiscriminada los servicios ecosistémicos proporcionados por los espacios naturales sin medir las consecuencias, de esta manera, gran parte de las decisiones del empresario frente a la crisis ambiental se originan por sus características culturales. 


\section{El empresario como actor social}

Las teorías administrativas buscan construir una cultura superficial. El sujeto es importante pero como medio para alcanzar los fines. Se crea una división estructural entre los individuos que toman decisiones y quienes obedecen, haciéndoles saber que si cumplen con las políticas implantadas lograrán alcanzar las metas, que no son otra cosa que obtener un alto rendimiento económico para un supuesto bienestar organizacional, que realmente es disfrutado por los altos ejecutivos, mientras que los subalternos solo se someten sin tener derecho a opinar, incluso contra su propia voluntad y su cultura, de la cual hablaremos más adelante.

Por otra parte, es importante destacar los cambios que han tenido las teorías administrativas dentro de su evolución y cómo influyen en el accionar de los actores empresariales, en este sentido Muñoz (2010, p. 245) indica:

Esta es la lógica de las modas administrativas, que por lo general rechazan a sus predecesoras, y además desconoce todo modo, estilo, método o modelo que la empresa haya utilizado en el pasado. Su visión es ahistórica, y adicionalmente su pretensión de universalidad la hace ciega a lo particular, lo local y lo cultural.

Es decir, las corrientes administrativas, a través del tiempo, se van sustituyendo unas con otras, lo cual hace que se pierda la continuidad o historia del conocimiento adquirido, solo se desarrollan de acuerdo a las condiciones que presenta el entorno y van de la mano con la evolución de las necesidades que revele la sociedad empresarial. La construcción de la definición del empresario se desenvuelve en un contexto meramente moderno que se ajusta a algo momentáneo.

Partiendo de lo anterior se identifica el antagonismo existente en lo que realmente define al hombre o empresario, según Hernández (2000, p. 20): "el hombre es por naturaleza un ser histórico y cultural particular, y no puede hacerse caso omiso de su circunstancia en aras de una abstracción y universalidad supuestamente científicas". 
La verdadera definición del hombre se construye a partir del estudio humanístico, valorativo y sustancial del ser humano como un ser racional con un pensamiento único que lo hace diferente e independiente, el cual está inmerso dentro de una sociedad y por pertenecer a ella, todas sus acciones y decisiones cumplen con un papel muy importante para el sostenimiento y crecimiento del contexto donde interactúa. Así mismo sucede dentro de las organizaciones, los hombres que administran los negocios cumplen con un perfil específico del cual dependen el fracaso o el éxito de la organización.

Para Marcela Hernández (2000) el empresario es un sujeto social, el cual debe adoptar diferentes roles dentro de la compañía, todos encaminados a la elaboración de estrategias que le permitan tomar sus propias decisiones con la responsabilidad de actuar con diligencia en el momento en que la organización requiera algún tipo de reestructuración.

\section{La cultura organizacional desde la formación del empresario}

La cultura organizacional permite identificar las diferencias que existen entre una y otra empresa. A pesar de tener las mismas características de constitución son administradas por distintos individuos con variadas formas de percibir e interpretar las cosas que lo rodean y con oposiciones en su actuar como sujetos sociales y sus objetivos a alcanzar dentro de la organización. La cultura organizacional es una base de construcción, determinada por el comportamiento, las vivencias y creencias de sus dirigentes, quienes la llevarán al fracaso o al éxito gracias a los rasgos propios de cada individuo. Pero, ¿cómo se percibe el significado de cultura?

La cultura relaciona al individuo y a la sociedad, por lo que para Rodríguez (2009, p. 69): "la cultura se desarrolla en cualquier comunidad de individuos que interactúan y se relacionan al perseguir objetivos, intereses, creencias, valores, rutinas y necesidades compartidas". Aspecto que determina la importancia de las interacciones sociales, así, la cultura es 
la interrelación existente entre los diversos sujetos con las características propias de cada comunidad dadas en el transcurso de la historia y demarcada por su ubicación geográfica, sus creencias, hábitos, constituciones familiares; las verdades que se contradicen con las de otras comunidades (Harvey, 1990). Se podría afirmar que la cultura está inmersa en cada grupo, comunidad, pueblo, sociedad, país, colonia. En cada espacio y estructura de conocimiento que nos hace diversos y únicos.

Esta interrelación permite que la cultura sea vista como un "sistema de normas y valores" Hernández (2000 p. 5), interiorizada por los actores de manera directa, sin mediaciones, y asumiéndola como una estructura independiente de los sujetos y no como parte constitutiva de estos, es decir, no es algo innato en el individuo sino creado por normas y valores que se forman en el transcurso de su historia. Para el enfoque desde las sociedades, la cultura es vista como resultado de categorías económicas de consumo, distribución y producción, determinadas por una perspectiva económica que afecta la caracterización de la cultura del individuo.

A partir de estas consideraciones, Hernández (2000) determina que los rasgos culturales de una organización están basados principalmente en los valores que forman el corazón de la cultura, es decir, en la moral del individuo, en su forma habitual y tradicional de pensar y hacer las cosas que la integran. Cada uno aporta para la conformación de la cultura organizacional, por tal razón, son características propias de la empresa.

La cultura enmarca diversas ópticas de desarrollo dentro de la organización, una de ellas es la responsabilidad social empresarial (RSE) como factor determinante de la cultura organizacional. Desde tiempo atrás se ha creído que la responsabilidad del empresario es generar riquezas y maximizar beneficios, pero este término es errado, dado que la RSE va mucho más allá de lo económico; va en el aporte que la empresa puede realizar al beneficio social y de sus stakeholders, así "la empresa debe ser principalmente un ente diseñado para satisfacer necesidades humanas con calidad" de acuerdo con Hamburguer (2007 p. 111) quien explica que la empresa deberá generar 
incremento en sus utilidades y, dentro de esta cultura, producir cambios para lograr el beneficio social y económico.

Es así como la responsabilidad social se enmarca en tres fases que Hamburguer (2007) clasifica así: la primera, "niveles de prácticas operativas" en las cuales el empresario se muestra indiferente ante los temas de responsabilidad; si no hay leyes que cumplir para evitar ser castigados no se hará nada para hacer un cambio en las prácticas gerenciales. La segunda, "nivel de prácticas eficientes", que corresponde a un cumplimiento moderado en donde adoptan las buenas prácticas pero no dan más de lo que se les exija. Y la tercera, "nivel de prácticas responsables" donde todos los empresarios deberían estar y los stakeholders exigir, donde no solo se actúa por las normas sino por convencimiento de que lo que se está haciendo es para beneficio de todos y no de unos pocos.

La cultura organizacional está dada, entonces, por cada individuo que la integra y por cada característica propia creada desde la educación: conocimientos, experiencias, gustos, valores, costumbres que lo hacen único y responsables o no, ante la sociedad y sus terceros.

Dentro de la responsabilidad social está inmerso el tema del manejo y cuidado del medio ambiente como generador de vida y riquezas, por tal razón en un próximo apartado se abordará el tema ambiental como factor determinante para la toma de decisiones gerenciales y la elaboración de estrategias para la sostenibilidad de la empresa.

\section{Territorio, empresa y región}

A partir de la concepción de la RSE, y desde los estudios de Hamburguer (2007), se determina que moverse dentro de la filosofía de la RSE se logra únicamente a través de alianzas estratégicas, es decir, que se deben crear redes dentro de los territorios. La noción de solución a lo ambiental se da solo por una comprensión holística de la problemática, en donde la idea de territorio se hace fundamental. 


\section{Las concepciones del territorio}

El concepto de desarrollo local debe entenderse como un proceso de crecimiento de una industria o una agregación de las empresas locales y un proceso de desarrollo territorial basado en la utilización sostenible de los recursos tangibles e intangibles presentes en un territorio determinado, que también incluye el ámbito social y cultural y la capacidad de autoorganización de los sujetos.

En cuanto a la tierra, o mejor dicho, el patrimonio territorial, no tanto redefinido como constitutivas de un "ser" como "ser", el objetivo es centrarse en las trayectorias de transformación, los elementos de continuidad, la estabilidad y la unidad diferencial en los personajes de contextos (Hanson, 2010). En estos modos, la descripción del patrimonio territorial es equivalente a la descripción del proceso de "recogida selectiva", a través del cual uno se identifica y le dice que no es tanto lo que queda del pasado, sino más bien lo que es reactivado en el presente, este sentido es lo que Marc Auge (1993) denomina los no lugares, espacios construidos fuera de los espacios formales pero estructurados desde aspectos que generan identidad, poder y pertenencia.

Estas premisas nos llevan a considerar que la identidad colectiva de los sujetos no solo se define sobre la base de su proximidad espacial, que no ha sido creada para compartir de forma pasiva un determinado territorio y sus significados simbólicos, sino que se deriva de la actuación colectiva de los individuos como portadores de prácticas y conocimientos, constructores del territorio y de las nuevas lógicas de referencia de la identidad de los lugares (Mercier, 2009).

De esta manera la interacción entre sujetos y lugares (entre los actores y el territorio) se construye mutuamente y en referencia a diferentes concepciones del territorio. Recordando que el territorio delimita reglas y comportamientos para ingresar a él, que se localiza y se estructura simbólicamente. El lugar es el simple espacio. Así, desde Mercier (2009), esta acción reconoce un territorio administrativo del área de conocimiento, un 
concepto relacionado con la composición y estructura dada a los lugares (el territorio como patrimonio o legado del pasado), y por último, una concepción del territorio como una construcción social que establece la identidad local en relación con la acción colectiva de los sujetos, en este caso de los empresarios, y vinculando con el apartado anterior, la noción de territorio del empresario estará sujeta a las afinidades culturales y al campo de poderes en el manejo de condiciones mínimas de producción. Desde lo ambiental, la RSE deconstruye una nueva noción de empresa, de territorio, de identidad cultural y de producción.

\section{Lo ambiental y la construcción empresarial}

La economía creciente ha construído un empresario capitalista que en el transcurso de la historia y según su cultura (como se observó en el primer apartado), permite que su análisis y toma de decisiones para el crecimiento empresarial se realice según la clásica estructura económica y política, dejando de lado su responsabilidad social, especialmente en lo concerniente al cuidado y manejo de los recursos naturales que afecta con su producción; y que han hecho que la ciencia económica se olvide de lo no económico, como afirman Morin y Kern (1993), es decir, han dejado a un lado lo social, lo cultural, el entorno, para convertirse en generadores de riquezas a través de la explotación natural y social.

El sistema ha tomado estos recursos para maximizar beneficios y generar desarrollos industriales, causando expansión urbana, contaminaciones, envenenamiento de suelos, urbanización masiva de regiones ecológicamente frágiles, deforestaciones, inundaciones, etc., en este sentido, ha olvidado que la economía depende de lo que depende de ella, señalan Morin y Kern (1993). Este afán desmesurado de obtener lucro no posibilita que el empresario tenga un pensamiento paralelo frente a su responsabilidad social y ambiental, sus diversos stakeholders y hacia el manejo desmesurado de los recursos naturales. 
La necesidad de originar responsabilidad social empresarial pide a gritos cambios desde la visión del empresario, ser éticos en las decisiones frente al tema ambiental, frenar la dilapidación de los recursos naturales que se convierte en la agonía del planeta. El empresario tiene que dejar de ser una empresa privada para convertirse en una empresa pública, es decir, generadora de beneficios para la sociedad a través de la retribución y la compensación al legítimo dueño por el consumo de recursos, y así formar un ciudadano corporativo, como afirma Carrillo (2006). Entonces se encuentra el empresario con un dilema ético de maximización de utilidad para el crecimiento económico o transformaciones estructurales en la organización para la conservación del medio ambiente que conlleven a la sostenibilidad.

El empresario está enmarcado en dos vertientes ambientales que son conocidas como Light Green y Dark Green; en el primero, las exageraciones del problema ambiental han sido fundadas por activistas y por los medios de comunicación, no reconoce que hay una crisis mundial; los individuos se limitan a cumplir solo leyes y normas, se afirma que realizan actividades ambientales siempre y cuando no afecten sus utilidades, es decir, donde el costo no supere el beneficio; "creen que los gastos ambientales están creciendo rápidamente y ofrece pocas posibilidades de recuperación económica" (Roth y Keller, 1997, p. 21). Los Dark Green reconocen y aceptan que hay una crisis ambiental, que es necesario realizar cambios desde la producción y para la producción, lo cultural, ética empresarial, y de forma consciente "reconocen los impactos que su actividad económica genera al presente y al futuro de las generaciones" (Roth y Keller, 1997, p. 23), de igual manera, aclara Montuschi (2002, p. 24), "las empresas deciden voluntariamente contribuir al logro de una sociedad mejor y un medio ambiente más limpio".

Desde esta clasificación podemos observar que la noción de territorio enmarcará entradas y salidas, como las explicadas por Mercier (2009), a partir de sus creencias, en los comportamientos frente a lo ambiental, las nociones de identidad y la conducta cultural, mencionadas. 


\section{Redes, empresarios y decisiones}

A partir de autores de geografía económica como Watts (2005) se logra develar cómo la construcción social puede verse desde una perspectiva de los estudios sociales de la ciencia. Traer a Watts aplica para el concepto de red, de red sociotécnica, según Bruno Latour (1998). Es importante destacar que en el propio concepto de red todavía no hay un consenso, no es tan fácil lograr definirlo espacialmente como "lugar" o "región" debido a que su naturaleza es dinámica, es parte de acciones e interacciones y no debe confundirse con las escalas espaciales. Se convierte en una serie de híbridos entre los actores humanos y no humanos, dándole agencia a unos y a otros (Watts, 2005), dejando de lado las simples tendencias relacionales estructuradas en un plano euclidiano.

La red tiene una multiplicidad de contenidos que enriquece el conjunto agregado de significados al concepto y permite un completo análisis epistemológico. El geógrafo brasileño Milton Santos (1996) determina que una definición más exacta y precisa de red se presta para ambigüedades, sin embargo, el mismo Santos (1996) logra develar que lo polisémico se permite más como una ventaja, y no habría ningún pensamiento diferente en el campo de la geografía, la ciencia se utiliza para manejar múltiples significados complejos y a veces antagónicos.

Con base en el ámbito de lo material, y siguiendo lo estructurado por Mercier (2009), se pueden apreciar las redes desde una plataforma de redes técnicas (energía, transporte, telecomunicaciones, infraestructura urbana, etc.), sin las que sería posible que el movimiento y las comunicaciones requeridas para el establecimiento de relaciones propias de la composición de otras redes existiese (políticas, simbólicas, culturales o en sentido estricto, "sociales"). No obstante, la técnica básica no es capaz de explicar los procesos relacionados con la forma de observación primaria, sino que es un resultado de las acciones que son propias a los procesos sociales (Bijker, 1987) y Latour, 1998). Con estos términos se derivan otros elementos esenciales: "la política", no solo el Estado, sino los diversos actores estratégicamente 
situados en el espacio mediante el establecimiento de territorialidad que revelan y superponen acciones diferentes, complementarias y contradictorias.

Esto no quiere decir que el lugar y la región se reduzcan a escalas espaciales, sino que tradicionalmente se analizan desde la perspectiva de zona, a menudo relacionada con la extensión espacial; por ejemplo, la idea de la región insertada entre lo global y lo local. En este sentido, Bijker (1987) explica que las relaciones de poder estructuran aspectos que pueden generar negociaciones y traducciones, siendo lo ambiental, un nuevo aspecto dentro de la construcción de la red, y el discurso de la RSE, una estructura sociotécnica que permitirá articularla, generando que la red se encuentre en los mismos parámetros, sociotécnicos y técnicopolíticos.

Desde lo estructurado por Watts (2005) y desde Mercier (2009) en la geografía, y cruzando con los sociólogos de la ciencia Latour (1998) y Bijker (1987) podemos definir algunos puntos importantes en cuanto a los estudios geográficos en relación con los empresarios: la red no debe ser tomada simplemente como un conjunto de líneas y puntos, sino como relaciones que se producen en el tiempo y el espacio de forma discontinua, es decir, sin necesidad de completar todos los nodos que la componen, estableciendo que se permite aliviar los cuellos de botella causados por las redes técnicas como las redes físicas (no siempre que no existe la tecnología se generan asociaciones, pero también la ausencia de la misma generan disgregaciones); un segundo aspecto es que toda la red geográfica está impregnada de la presencia de aspectos técnicos (Latour, 1998), de acciones políticas definidas por las personas o grupos (Hernández, 2000) y que conlleva a estructuras socioespaciales llenas de contradicciones (Mercier, 2009).

\section{Redes y desarrollo regional}

Dentro del estudio de los empresarios también se encuentra la región como el contexto en el cual se desenvuelve la organización, estudiarla implica comprender todo su entorno, lo que la caracteriza, lo que la diferencia de las demás; estas definiciones constituyen las costumbres, los hábitos, los valores 
y las oportunidades de generar empresa en cuanto al tipo de producción, comercialización y/o servicio; la importancia de la familia, la manera en que socializan, los tipos de tierras, el clima, la población, la geografía, la política; son cualidades que construyen la cultura de la nación.

El profesor Milton Santos en su capítulo sobre redes espaciales (1996) ayuda a explicar lo regional a través de la dialéctica en el territorio de la relación global-local, que establece a través de la atracción de hacer un contraste entre "una empresa local y la sociedad nacional, el territorio y el territorio nacional, local, e incluso un sitio de formación económico-social (o regional) y la formación socioeconómica nacional” (Santos, 1996, p. 216).

Entonces, ¿cómo hablar de estos temas? La red sería, pues, el punto de encuentro entre estas realidades, ya que el movimiento social incluye aspectos dinámicos de localidades cercanas y lejanas, universales, impulsado por grandes organizaciones; la realidad de la dualidad que se origina cerca-lejos, que se puede cerrar y estar ausente, así como lo que es distante puede convertirse en cercanía. Al mismo tiempo, las redes globales y locales también son una y son múltiples. En este sentido Santos (1996) logra explicar que las nociones de desarrollo regional y desarrollo local se dan por las aglomeraciones de empresarios en torno a fines y medios, pero también a afinidades culturales, políticas y económicas, de esta manera, las redes no son estáticas, se mueven, se sitúan, se posicionan, como diría Bourdieu (1994), en el campo, generan acciones de poder y de acción. Si esperamos lograr un empresario encaminado hacia el desarrollo sustentable es necesario estudiar las redes ya formadas, las interacciones local-global, empresario-cluster, ciudad-región.

\section{Reflexiones finales}

Las redes permiten transmitir estructuras culturales. Comprendido que el paradigma ambiental es parte de la red, el discurso de la RSE es un nodo sociotécnico, y la construcción de discusiones alrededor de la misma son las traducciones que permiten este desarrollo del factor del empresario 
desde los no lugares de Auge (1992), en los espacios de entrada y salida que explica Mercier (2009).

Esta cultura es transmitida a la organización, y empleada por todos los miembros de la empresa, lo cual hace que la organización adquiera su propia cultura, "por ejemplo en Chile se discute sobre la falta de un espíritu empresarial, mientras que en Colombia se resalta el carácter emprendedor de los empresarios de la región de Antioquia" (Twinam, 1985 y Patiño, 2003 citados por Hernández, 2000).

Ejemplos como el anterior demuestran la realidad y la diversidad cultural que se puede presentar en Colombia, por lo cual no se debe generalizar la manera en que razonan todos los empresarios del país, al inferir que la estructura geográfica de Colombia está compuesta por diversidad regional, más desde las tesis de regiones culturales explicadas por Orlando Fals Borda (1990) que desde la perspectiva clásica o idílica, y a su vez por espacios que difieren su composición cultural creando redes y procesos diferenciados. El pequeño empresario latinoamericano debe buscar mecanismos que le permitan consolidar una estructura sólida de su organización, afianzando sus lazos institucionales con todo el equipo que conforma su contexto empresarial, a nivel interno y externo instaurando nuevos campos de investigación desde la relación entre ciencias sociales y escenarios empresariales. 


\section{Referencias bibliográficas}

Auge, M. (1992). Los No lugares espacios del anonimato. Antropología de la posmodernidad. Barcelona: Ed. Gedisa.

Bijker, W. E., Hughes, T. P., y Pinch, T. J. (Eds.). (1989 (1987)). The Social Construction of Technological Systems: New Directions in the Sociology and History of Technology. Cambridge, Massachusetts: The MIT Press.

Bourdieu, P. (1994). Razones prácticas sobre la teoría de la acción. Paris: Editions de Seuil.

Carrillo, J. (2006). Ética y empresa, ¿son compatibles la ética y la gestión empresarial en el contexto de la economía de mercado? Bogotá: Ediciones Universidad Nacional de Colombia, Facultad de Ciencias Humanas-Unibiblos.

Cendejas, G., Hernández, G. (2000). Los nuevos ejes de la cultura organizacional de las empresas e instituciones en el siglo XXI. Recuperado de http://www. razonypalabra.org.mx/actual/hernandezcendejas

De la Garza, E. (1998). Epistemología de los modelos de producción. Buenos Aires. Dupuis, J. (2010). El capitalismo: origen, esencia y variedad. En Sociología de la empresa Del marco histórico a las dinámicas internas. Bogotá: Siglo del Hombre Editores, Fondo editorial universidad EAFIT.

Fals Borda, O. (1990). Región y cultura: algunas implicaciones teóricas y políticas. Bogotá.

Hamburguer, A. (2007). Ética y responsabilidad social de las empresas. Recuperado de http://datateca.unad.edu.co/contenidos/101010/Evaluativas/ EvaluativaUnidad8/etica_y_RSE_enlaempresa.pdf

Hanson, S. (2010). Gender and mobility: New approaches for informing sustainability. Gender, Place, and Culture 17 (1): 5-23.

Harvey, D. (1990). La condición de la posmodernidad. Oxford: Ediciones Amorrotu. Hernández R, M. (2000). La perspectiva sociológica de los actores empresariales. En De la Garza, Tratado latinoamericano de sociología. México: El Colegio de México, Facultad Latinoamericana de Ciencias Sociales, Fondo de Cultura Económica.

Latour, B. (1998). La tecnología es la sociedad hecha para que dure. En Domenech y F. Tirado, Sociología simétrica. Ensayos sobre ciencia, tecnología y sociedad. Barcelona: Gedisa. 
Mercier, G. (2009). Hacia una teoría del lugar. En Geografía humana. Lecturas en teoría de la geografía, ed. Jhon Williams Montoya G. 21-40. Bogotá: Universidad Nacional de Colombia, sede Bogotá, Facultad de Ciencias Humanas, Departamento de Geografía.

Montuschi, L. (2002). Ética y razonamiento moral. Dilemas morales y comportamiento ético en las organizaciones. Buenos Aires: Cuadernos de trabajo.

Morin, E., Kern, A. (1993). La agonía planetaria. Paris: Editions de Seuil.

Muñoz, G., (2010). Cambio y cultura organizativa, En Sociología de la empresa. Del marco histórico a las dinámicas internas. Bogotá: Siglo del Hombre Editores, Fondo editorial universidad EAFIT.

Pérez, R. (2007). Estructura y cultura organizacional en la Pyme colombiana: Análisis en empresas bogotanas. Recuperado de http://bibliotecadigital. univalle.edu.co/bitstream/10893/2154/1/estructura\%20y\%20cultura\%20 organizacional.pdf

Prieto, J. (2008). Gestión estratégica organizacional. Guía práctica para el diagnóstico empresarial. Bogotá: Ecoe Ediciones.

Rodríguez, R. (2009). La cultura organizacional, un potencial activo estratégico desde la perspectiva de la administración. Recuperado de http://www.redalyc. org/pdf/877/87722106.pdf

Roth, H., Keller, C. (1997). Quality Profits and the Environment: Diverse Goals of Common Objectives? tomado de http://www.imanet.org/

Santos, M. (1996). A Natureza do Espaço. Técnica e tempo, razão e emoção. São Paulo: Editora Hucitec.

Solomon, R. (1995). La ética de los negocios. Singer, P. Compendio de ética. Madrid: Alianza Editorial.

Watts, M. (2005). Nature: Culture en Spaces of Geographical Thought: Deconstructing Human Geography's Binaries. Editado por Paul Cloke y Ron Johnston. Sage Pub. Londres. 